\title{
A Technical Tip for Ensuring Parallel Screw Fixation of the Medial Malleolus
}

\author{
Edward Yates $^{1 *}$, Giles Foley ${ }^{2}$ and Christopher Thomas ${ }^{2}$
}

${ }^{1}$ Department of Orthopaedics, East Lancashire NHS Foundation Trust, Royal Blackburn Hospital, Blackburn, Lancashire, BB2 3HH, UK ${ }^{2}$ Department of Orthopaedics, East Lancashire NHS Foundation Trust, UK

\begin{abstract}
Parallel screw alignment can be challenging. We describe a simple, reproducible technique for the fixation of the medial malleolar fragment with two parallel screws, using a $1.6 \mathrm{~mm} \mathrm{~K}$-wire and the small fragment $\mathrm{AO}$ drill-guide.
\end{abstract}

Keywords: Ankle; Fracture fixation; Internal

\section{Introduction}

Medial malleolus fractures with more than $2 \mathrm{~mm}$ of displacement are associated with a non-union rate of 5-15\% [1]. Surgical fixation of these fractures is considered standard practice in Orthopaedic trauma surgery. Whilst single screw fixation is an option, the AO Foundation recommend two parallel, partially threaded cancellous screws to prevent rotation of the fragment [2]. In order to maximise interfragmentary compression, the screws should be placed perpendicular to the fracture plane [2]. Achieving parallel screw alignment can be challenging.

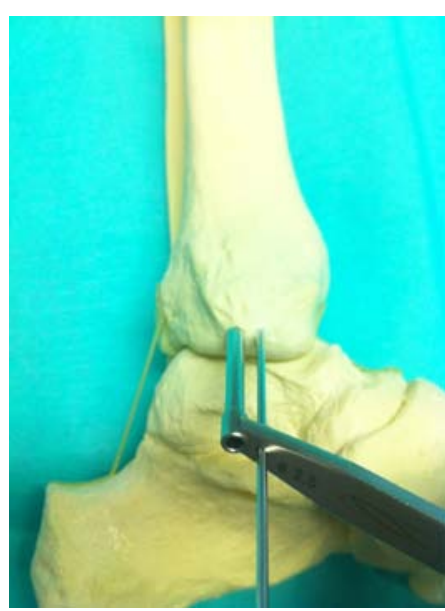

Figure 1: AO Small Fragment Drill-guide mounted over the $1.6 \mathrm{~mm} \mathrm{~K}$-wire using the aperture adjacent to the $2.5 \mathrm{~mm}$ drill sleeve.

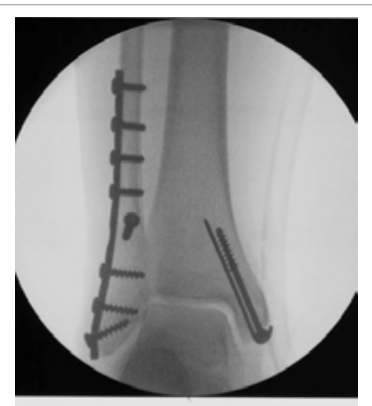

Figure 2: Mortise view radiograph demonstrating parallel internal fixation of the medial malleolar fragment. Note the combined use of K-wire and partiallythreaded cancellous screw.

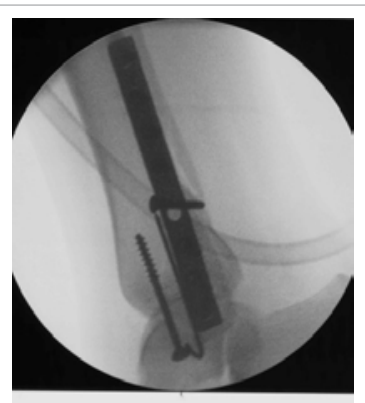

Figure 3: Lateral view radiograph demonstrating parallel internal fixation of the medial malleolar fragment.

\section{Technique}

Following anatomical reduction, a $1.6 \mathrm{~mm}$ smooth $\mathrm{K}$-wire is passed through the centre point of the malleolar fragment and into the tibial metaphysis perpendicular to the plane of the fracture. Once applied, we advise fluoroscopic assessment to ensure that the K-wire is in an adequate position and not penetrating the joint. The small fragment $\mathrm{AO}$ drill-guide has an aperture adjacent to the $2.5 \mathrm{~mm}$ drill sleeve that is oriented parallel to the sleeve itself (Figure 1). When mounted over the guidewire using the described aperture, the drill-guide can then be rotated around the axis of the K-wire facilitating parallel drill holes that are perpendicular to the fracture plane (Figures $2 \& 3$ ). In cases where the malleolar fragment is too small to accommodate two screws, the initial stabilising K-wire can be eccentrically placed and left in situ to prevent rotation (Figures $2 \& 3$ ).

\section{Discussion}

Parallel screw alignment can be challenging. In our department

*Corresponding author: Edward W Yates, Specialist Registrar in Trauma and Orthopaedics, Royal Blackburn Hospital, Haslingden Road, Blackburn, BB2 3HH, UK, Tel: 01254736 029; Fax: 01254293 512; E-mail: eddyates@doctors.org.uk

Received November 19, 2011; Accepted January 02, 2012; Published January 10, 2012

Citation: Yates E, Foley G, Thomas C (2011) A Technical Tip for Ensuring Parallel Screw Fixation of the Medial Malleolus. J Trauma Treat 1:106. doi:10.4172/21671222.1000106

Copyright: (c) 2011 Yates E, et al. This is an open-access article distributed under the terms of the Creative Commons Attribution License, which permits unrestricted use, distribution, and reproduction in any medium, provided the original author and source are credited. 
Citation: Yates E, Foley G, Thomas C (2011) A Technical Tip for Ensuring Parallel Screw Fixation of the Medial Malleolus. J Trauma Treat 1:106. doi:10.4172/2167-1222.1000106

Page 2 of 2

this technique has improved our ability to stabilise the medial malleolar fragment with perfectly placed parallel screws. Importantly, we have found this an easy, safe and reproducible technique. In addition, our technique avoids the need for a relatively expensive cannulated screw system, where the $\mathrm{K}$-wires are more flexible, less predictable and therefore difficult to place in parallel fashion.

\section{References}

1. Hamilton SW (2007) Parallel screw fixation of the medial malleolus. Ann R Coll Surg Engl 89: 729-730.

2. Ruedi TP, Buckley R, Moran CG (2007) AO Priniciples of Fracture Management (2ndedn) Thieme. 